\title{
Transciptome and Metabolomics Analysis of Pepper (Yin Chuan Cavel) Male Sterility Lind
}

Xiujuan Yan ${ }^{1} \quad{\text { Xin } \mathrm{He}^{2}}^{2}$ Yunxia Zhao ${ }^{1} \rrbracket \quad$ Jingxia Gao $^{1} \quad$ Xuemei Wang ${ }^{1}$

1 Institute of Germplasm Resources, Ningxia Academy of Agriculture and Forestry Sciences, Yinchuan Ningxia, 750002, China

2 General Station of Forest Pest Management and Quarantine of Ningxia, Yinchuan Ningxia, 750001, China

\Corresponding author Email: wxm92036h@163.com

Molecular Plant Breeding, 2020, Vol.11, No.24 doi: $10.5376 / \mathrm{mpb} .2020 .11 .0024$

Received: 25 Sep., 2020

Accepted: 27 Sep., 2020

Published: 20 Oct., 2020

Copyright (C) 2020 Yan et al., This article was first published in Molecular Plant Breeding in Chinese, and here was authorized to translate and publish the paper in English under the terms of Creative Commons Attribution License, which permits unrestricted use, distribution, and reproduction in any medium, provided the original work is properly cited.

Preferred citation for this article:

Yan X.J., He X., Zhao Y.X., Gao J.X., and Wang X.M., 2020, Transciptome and metabolomics analysis of pepper (Yin Chuan Cavel) male sterility lind, Molecular Plant Breeding, 11(24): 1-8 (doi: 10.5376/mpb.2020.11.0024)

\begin{abstract}
In order to study the relation of different gene expression and Metabolomics difference of pepper (Yin Chuan Cavel) male sterility lind pollen abortion. The transciptiome was sequenced using RNA-seq technology, the metabolomics was tested by UPLC-MS/Ms. The result was analyzed by GO categories and KEGG enrichment analysis. A total of 3319 different expression genes were successfully obained, include up genes 800, down genes 2 519. Of the 536 metabolites identified, a total of 102 metabolites were significantly differentiated. The differential metabolites involved up regulation metabolites 68 , regulation metabolites 34 . Included 17 amino acid derivatives, 16 Nucleotides and derivatives, 13 glyceryl ester, 10 sphingolipids, 8 flavonoids and phenolic acids, 6 in other classes (mainly related to carbohydrate metabolism). Differential metabolites and significant differential expression genes are mainly concentrated in protein related amino acid metabolism pathway and flavonoid metabolism pathway. The results showed that the significant differential expression of transcription genes, through the regulation of metabolism related enzymes, resulted in the differential metabolism of lipid, Phenolic acids and carbohydrate metabolites, resulted in pollen abortion of the Pepper (Yin Chuan Cavel) male sterile.
\end{abstract}

Keywords Pepper; Male sterility; Transciptome; Metabolomics

Pepper is a very important vegetable crop and loved by the Chinese. It is nutritious and can stimulate appetite, reduce blood pressure, improve human resistance (Cai et al., 2010). China is a big country of pepper cultivation, production and processing. In recent years, the planting area of pepper is increasing, and the output ranks first in the world. With the development of industry, the requirements of pepper breeding and seed production are also increasing (Suo, 2011). Seed production with male sterile materials is an effective method to simplify seed production procedures, reduce seed production costs, and ensure seed purity and quality. The correlation between transcriptome and metabolome of Pepper (Yin Chuan Cavel) Male Sterility Lind was studied, which has important theoretical significance for further understanding the pollen abortion mechanism of its male sterile line (Chen et al., 2011).

RNA-Seq method is widely used in plant differential gene expression analysis and new gene mining research, can effectively provide more information on biological transcription level (Trapnell et al., 2010; Liao et al., 2017; Qin et al., 2020). Transcriptome and metabonomics integration analysis refer to the normalization and statistical analysis of the batch data of different biomolecular levels from transcriptome and metabolomics, so as to establish the data relationship among different levels of molecules (Ding et al., 2019). Combined with functional analysis, metabolic pathway enrichment, correlation analysis and other biological function analysis, this experiment systematically and comprehensively analyzed the biological molecular function and regulatory mechanism of differentially expressed transcriptome in the anther of Pepper (Yin Chuan Cavel) Male Sterility Lind. Screened out the key metabolic pathways, differentially expressed genes and differentially accumulated metabolites (Chen et al., 2013). To further understand the mechanism of pollen abortion caused by differential gene expression regulating substance metabolism. 


\section{Results}

\subsection{Transcriptome sequencing and differential gene screening}

Total RNA was extracted from anther samples of dual-purpose lines for library preparation. The library was sequenced with Illumina HiSeq ${ }^{\mathrm{TM}}$ 2500. FDR and $\log _{2} \mathrm{FC}$ were used to screen the differential genes. The screening conditions were $\mathrm{FDR}<0.05$ and $\left|\log _{2} \mathrm{FC}\right|>1$ (Roberts et al., 2011). There were 35336 genes in the reference genome. Through comparison, the number of known genes detected in the male sterile line (K) and sterile line (B) of pepper (Yinchuan Cavel) was 23 768, accounting for $67.26 \%$ of the comparison genes. A total of 3319 genes were detected with significant difference, including 800 genes significantly up and 2519 genes significantly down.

\subsection{Different Gene (GO) Annotation}

Go functional classification annotation and significant enrichment analysis of differentially expressed genes in Pepper (Yin Chuan Cavel) Male Sterility Lind (Qin et al., 2020), K-VS-B (Figure 1): 230 genes down regulated and 965 genes up-regulated in metabolic process; 221 genes down-regulated and 863 genes up-regulated in cell process; 239 genes down-regulated and 691 genes up-regulated in catalytic activity; 211 genes down-regulated and 876 genes up-regulated in combining process. The differential genes were mainly concentrated in the process of metabolism, catalysis and metabolic regulation, which indicated that the differential gene expression of Pepper (Yin Chuan Cavel) Male Sterility Lind was significant in its metabolic activity, and the enrichment of differential genes involved in metabolic regulation was relatively rich.

\subsection{Metabonomics determination and analysis}

Anther samples were divided into fertile group and sterile group. 536 metabolites were detected in the anthers of fertile and sterile lines of pepper (Yinchuan Cavel) by UPLC-MS/MS detection platform. There were 102 differential metabolites, including 34 down regulated and 68 up regulated. As shown in Figure 2, the differential metabolites were divided into 19 groups, there are 17 amino acid derivatives, 16 Nucleotides and derivatives, 13 glyceryl ester, 10 sphingolipids, 8 Flavonoid and 8 Phenolic acids, 6 others (mainly related to sugar metabolism). RCA analysis of K-vs-B differential metabolites mix. Mix The quality control results are shown in Figure 3 . The detected differential metabolites are R2 $>0.93$, close to 1 , indicating that the results have high repeatability.

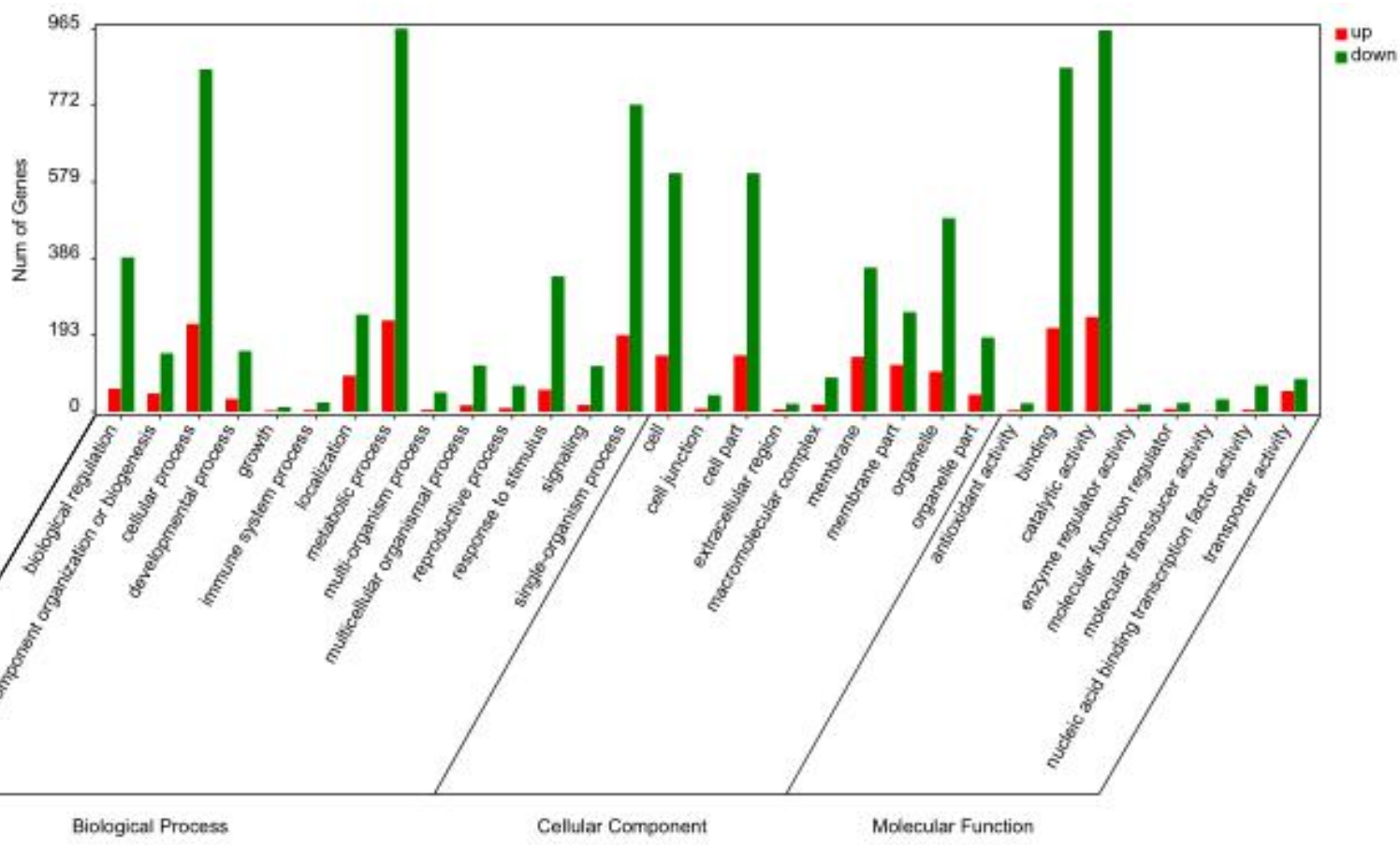

Figure 1 Differential gene GO terms of K-VS-B 
Molecular Plant Breeding 2020, Vol.11, No.24, 1-8

$\mathrm{http} / / /$ genbreedpublisher.com/index.php/mpb

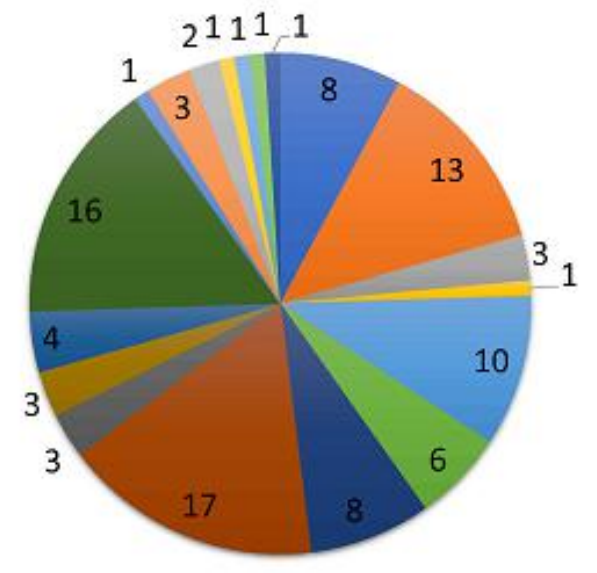
Elavonoid
a Flavonols
Alkaloids
Elavanols
- Free fatty acids
athers
- Phenolic acids
- Amino acids and derivatives $\mathbf{\text { u Plumerane }}$
Eihydroflavone
- Organic acids
- Nucleotides and derivatives
- Dihydroflavonol
alycerol ester
a Phenolamine
a Flavonoid carbonoside
asphingolipids
an Chalcones
Isoflavones

Figure 2 The distribution of diferent metabolites of K-VS-B

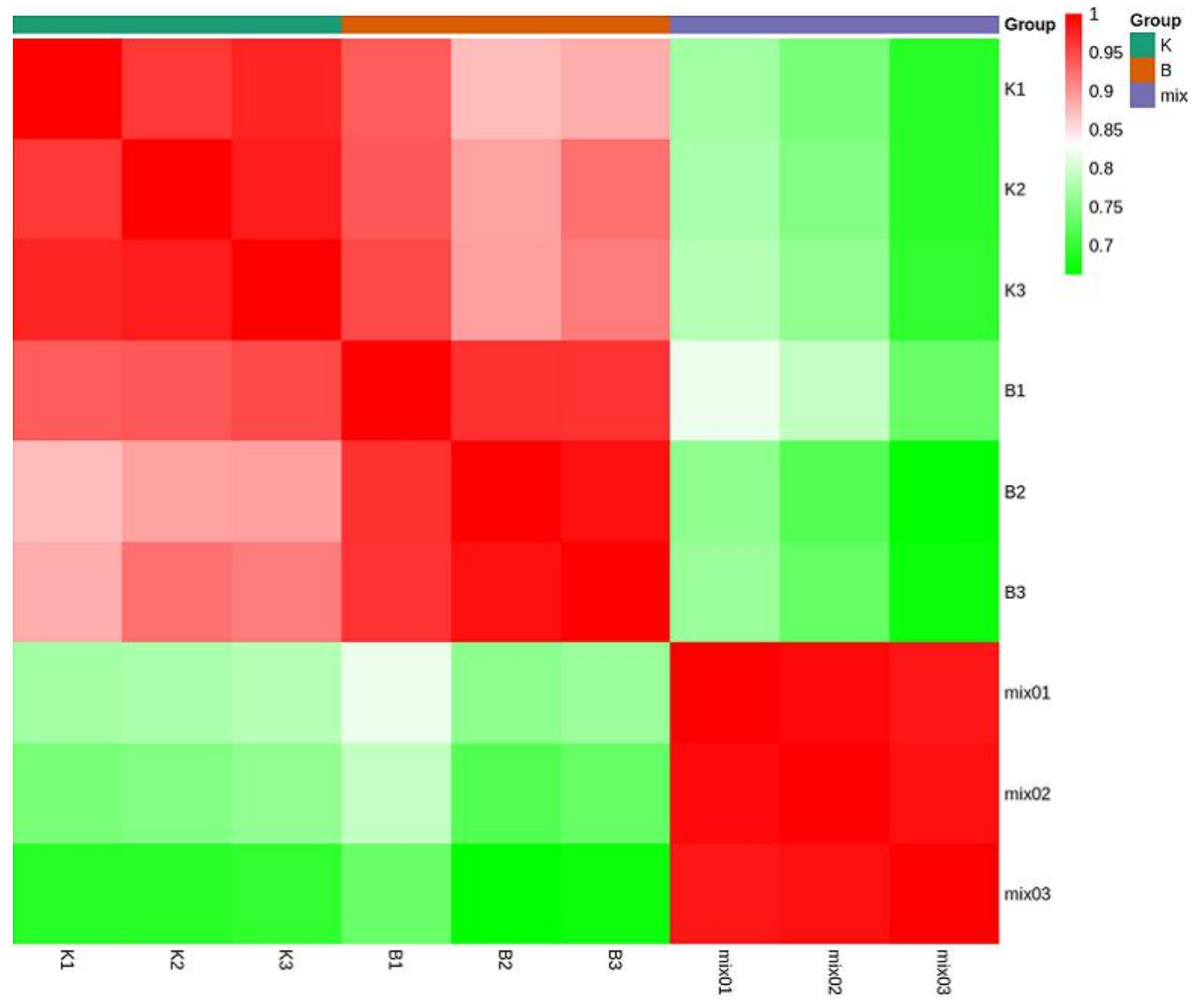

Figure 3 The RCA analysis and mix Mix,quality control sample K-VS-B 


\subsection{Correlation analysis between differential genes and different metabolites}

The cor program in $\mathrm{R}$ was used to calculate the Pearson correlation coefficient of genes and metabolites, and nine quadrant diagram was used to show the difference multiple of gene metabolites with Pearson correlation coefficient greater than 0.8 in each difference group. The results are shown in Figure 4: In quadrant 5, there was no differential expression between genes and metabolites; in quadrants 3 and 7, there was a positive correlation between gene expression and metabolites, and the change of metabolites might be caused by the positive regulation of genes; in quadrants 1, 2 and 4, there was correlation between metabolites and gene expression, and the up regulation of metabolites in quadrants 6,8 and 9 was negatively correlated with the expression of metabolites The metabolites were not changed or down regulated (Limin Sun, 2019). According to the correlation distribution of differential genes and different metabolites, there was significant correlation between differential genes and differential metabolites, which had a strong regulatory effect on anther metabolism of Yinchuan Yangjiao male sterile dual-purpose line.

\subsection{Analysis of differential genes and metabolites combined with KEGG}

Through KEGG enrichment analysis, we can find out the metabolic pathways with significant enrichment of differential genes and metabolites and show the enrichment degree of pathways with both differential metabolites and differential genes, so as to further understand the biological functions of differential genes (Kanehisa et al., 2013). The results showed in Figure 5: the enrichment pvalue value of differential expression genes and differential metabolites pvalue $<0.05$, differential expression is significant; however, it is not proportional distribution in the unified metabolic pathway, which indicates that not all differentially expressed genes regulate the metabolic pathway. The main metabolites were in Biosynthesis of amino acids, Pyrimidine metabolism, Tryptophan metabolism and Cysteine, methionine metabolism and Flavonoid biosynthesis. The results showed that the significantly differentially expressed transcriptional genes played a key role in the above pathways, resulting in the differential expression of the related products of protein metabolism and flavonoid metabolism in anthers, which affected the pollen abortion of Yinchuan Zanthoxylum annuum male sterile line.

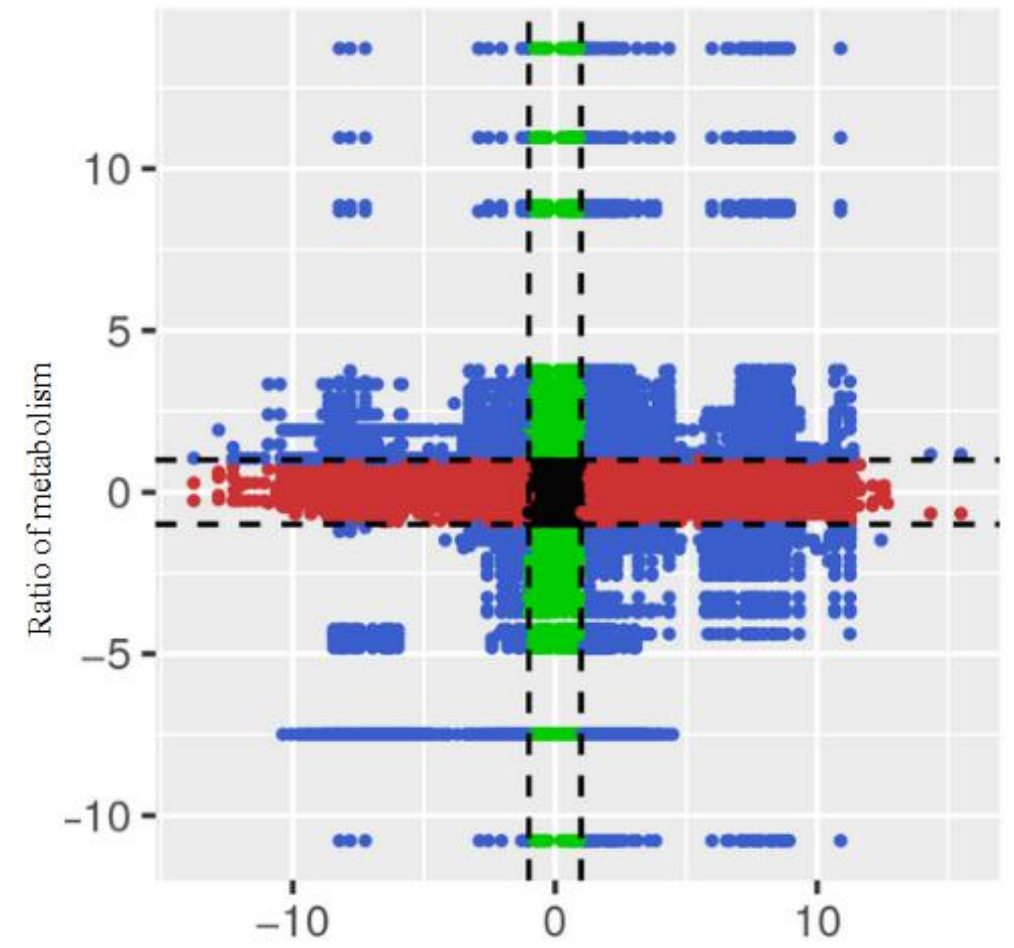

Ratio of gene

Figure 4 Nine quadrant of correlation between differential genes and differential metabolites 


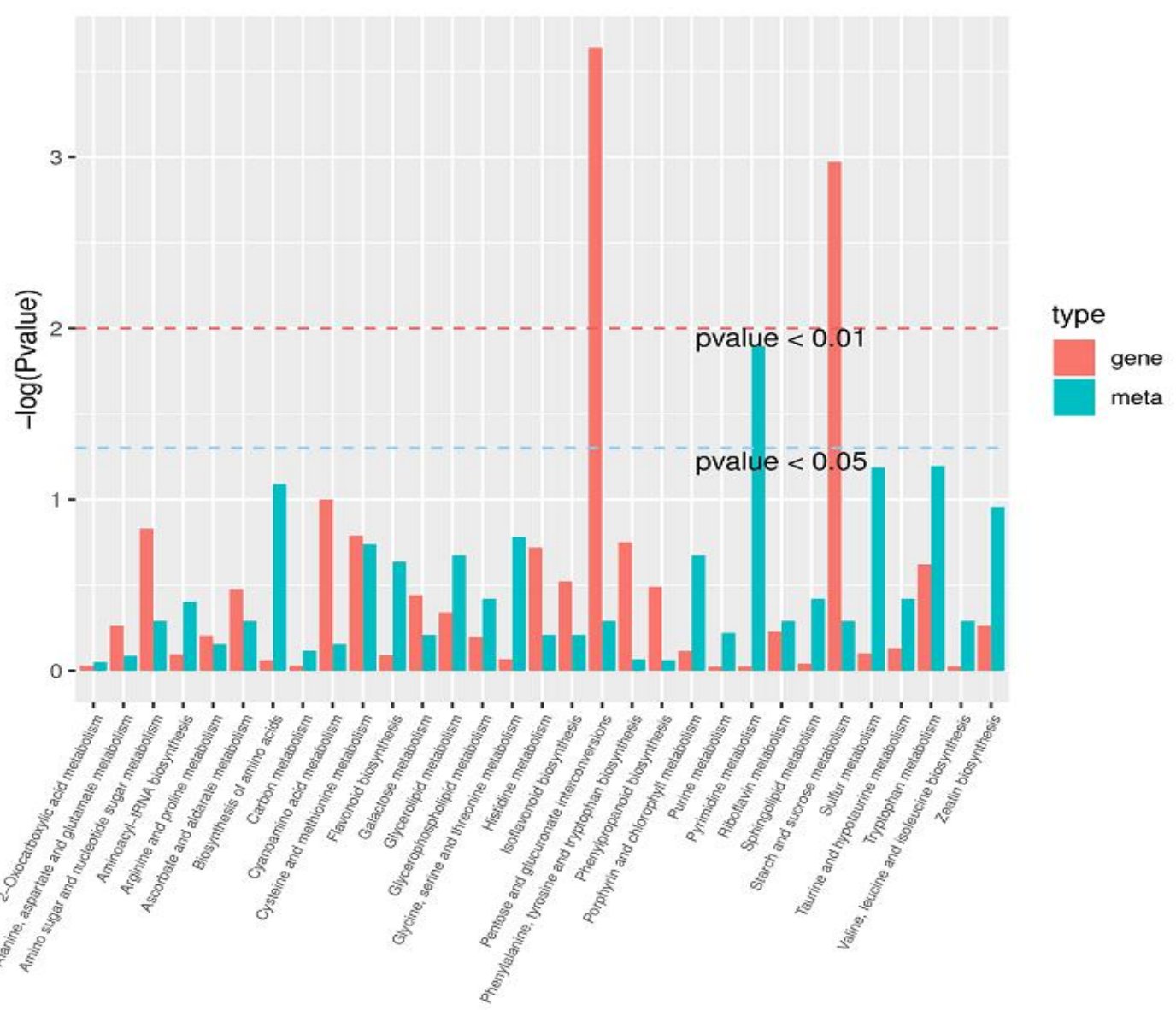

Figure 5 Analysis of pvalue histogram by enrichment of differential gene and differential metabolite KEGG

\subsection{Analysis of O2PLS}

All the differential genes and metabolites were selected to establish O2PLS model, and the important variables influencing the other omics were screened out. Due to the large number of genes and metabolites, only the top 10 genes and the top 10 metabolites that have great influence on the other omics are listed. The results are shown in Table 1. The top ten metabolites of differentially expressed genes were mainly glycosides, amino acids and phenolic acids. The results showed that the expression regulation of differential genes between male sterile lines and fertile lines resulted in significant differences in transcriptome, which resulted in significant regulation of material metabolism, especially glycoside energy metabolism related substances.

Table 1 Significant difference genes and differential metabolites

\begin{tabular}{llll}
\hline Metabolites ID & Chemical material & Gene ID & Length (bp) \\
\hline mws0913 & Trifolin & Capana10g000707 & 0.068908 \\
pme0088 & Luteolin & Capana01g003618 & 0.06124 \\
mws4176 & DL-Alanyl-DL-phenylalanine & Capana03g004625 & 0.058896 \\
mws0216 & Trans-4-Hydroxy-L-proline & Capana02g002479 & 0.056248 \\
pme0274 & 6-Aminocaproic acid & Capana00g003963 & 0.051817 \\
pme2433 & Diethanolamine & Capana03g000102 & 0.051389 \\
mws5042 & Glycylphenylalanine & Capana03g004566 & 0.051224 \\
mws0064 & Eriodictyol & Capana12g000531 & 0.051129 \\
mws0671 & L-Homoserine & Capana09g002327 & 0.050909 \\
mws0636 & Phe-Phe & Capana04g000466 & 0.049434 \\
\hline
\end{tabular}




\section{Conclusions}

The anther of Pepper (Yin Chuan Cavel) Male Sterility Lind analysis by RNA-Seq, compared with the reference genome, a total of 3319 genes were detected, which showed that the difference in transcriptome between male sterile line and fertile line was very significant. The results showed that the differentially expressed genes in Yinchuan Yangjiao pepper male sterile dual-purpose line played a regulatory role in its metabolic activities, and the accumulation of differential genes involved in metabolic regulation was rich (An et al., 2014; Trapnell et al., 2010). There were 34 down regulated and 68 up regulated differential metabolites detected in the anthers of fertile and sterile lines. The main metabolites were amino acid and derivatives, nucleotides and derivatives, glycerol ester, flavonoids, phenolic acids and sugar metabolism related substances ( $\mathrm{Lu}$ et al., 2020). Amino acids, nucleotides, flavonoids and sugars are all important substances affecting pollen development, the significant difference accumulation between fertile and sterile lines indicated that pollen abortion of male sterile lines was closely related to the differential metabolism and accumulation of the above substances. The results of transcriptome and metabolome analysis showed that the differential gene expression and metabolites in mature anthers of Pepper (Yin Chuan Cavel) Male Sterility Lind were mainly concentrated in the protein synthesis pathway of amino acid biosynthesis, pyrimidine metabolism, tryptophan metabolism, cysteine and methionine metabolism and flavonoid biosynthesis pathway. These results indicate that the significant differential expression of transcriptional genes in the above pathways plays an important role in regulating the accumulation of metabolites. The differential expression of transcriptional genes regulated the metabolism of key metabolic pathways, resulting in significant differential accumulation of glycosides and energy substances closely related to pollen development, which affected pollen development of male sterile lines and led to pollen abortion; The regulation of flavonoid metabolism is related to the scavenging of reactive oxygen species (ROS), which may lead to pollen abortion. It can be inferred that the differential expression of differential genes can regulate the metabolism and accumulation of lipid, fenac and carbohydrate metabolites related to pollen development, and then lead to pollen abortion (Li et al., 2016; Li et al., 2015).

\section{Materials and Methods}

\subsection{Materials}

The male sterile line of Yinchuan Yangjiao pepper was bred by pepper breeding research group of Germplasm Resources Research Institute of Ningxia Academy of agricultural and Forestry Sciences. It will be planted in May 2019. At the full flowering stage, the pollen abortion was identified by the acetic acid magenta staining microscope, and the fertile and sterile lines were identified. The mature anthers were sampled. The anthers of each sample were collected from ten materials and stored in the refrigerator at $-80^{\circ} \mathrm{C}$.

\subsection{RNA extraction and transcriptome sequencing}

After total RNA was extracted, eukaryotic mRNA was enriched by Oligo (dT) beads, while prokaryotic mRNA was enriched by removing rRNA by Ribo-Zero TM Magnetic Kit (Epicentre). Then the enriched mRNA was fragmented into short fragments using fragmentation buffer and reverse transcripted into cDNA with random primers. Second-strand cDNA were synthesized by DNA polymerase I, RNase H, dNTP and buffer. Then the cDNA fragments were purified with Qia Quick PCR extraction kit, end repaired, poly (A) added, and ligated to Illumina sequencing adapters. The ligation products were size selected by agarose gel electrophoresis, PCR amplified, and sequenced using Illumina HiSeq TM 2500 by Gene Denovo Biotechnology Co. (Li et al., 2020; Limin Sun, 2019). Three replicates were set for each sample, and the extraction and sequencing were entrusted to Gene Denovo Guangzhou Biology Limited.

\subsection{Extraction and analysis of metabolites}

The freeze-dried anther was crushed using a mixer mill (MM 400, Retsch) with a zirconia bead for 1.5 min at 30 Hz. $100 \mathrm{mg}$ powder was weighted and extracted overnight at $4^{\circ} \mathrm{C}$ with $0.6 \mathrm{ml} 70 \%$ aqueous methanol. Following centrifugation at $10000 \mathrm{~g}$ for $10 \mathrm{~min}$, the extracts were absorbed (CNWBOND Carbon-GCB SPE Cartridge, 250 mg, $3 \mathrm{ml}$; ANPEL, Shanghai, China, www.anpel.com.cn/cnw) and filtrated (SCAA-104, $0.22 \mu \mathrm{m}$ pore size; 
ANPEL, Shanghai, China, http://www.anpel.com.cn/) before UPLC-MS/MS analysis. The experiment was completed by Wuhan Metware Biology Limited.

\subsection{Data analysis method}

Transcriptome and metabolomic data were annotated to GO database (http://www.geneontology.org/). The gene number of each term was calculated, and the functional classification statistics were carried out by using WEGO software. The correlation between the detected genes and metabolites was analyzed, and the Pearson correlation coefficient of genes and metabolites was calculated by COR program. Using KEGG database (http://www.genome. $\mathrm{jp} / \mathrm{kegg} /$ ). The pathway enrichment of different genes and metabolites was analyzed. O2PLS analysis was used to explore the association between differentially expressed RNA and different metabolites. O2PLS model is used to analyze the integration between the two data sets, including the relationship between systems biomics, molecular regulatory mechanism phenotype correlation. Through O2PLS model, we can not only obtain the correlation coefficient between variables, but also obtain the weight of variables in the model, so as to find the key regulatory phenomena more accurately (Chen et al., 2013; Roberts and Pimentel, 2011).

\section{Authors' contributions}

In this study, Yan Xiujuan is responsible for data analysis and paper writing of experimental design; He Xin is responsible for running point and sampling work; Gao Jingxia and Zhao Yunxia are responsible for assisting data analysis and paper revision; Wang Xuemei is responsible for guiding experiment and paper writing. All authors read and agree to the final text.

\section{Acknowledgment}

This research is jointly funded by the natural fund project of Ningxia Hui Autonomous Region (2019AAC03156) and the whole industry chain innovation demonstration project of Ningxia Academy of agriculture and Forestry Sciences (QCYL-2018-03).

\section{References}

An H., Yang Z.H., Yi B., Wen J., Shen J.X., Tu J.X., Ma C.Z., and Fu T.D., 2014, Comparative transcript profiling of the fertile and sterile flower buds of pol CMS in B. napus, BMC Genomics, 15: 258

https://doi.org/10.1186/1471-2164-15-258

PMid:24707970 PMCid:PMC4051170

Anthocyanin Changes in Red Maple (Acer rubrum) Leaves, SCIENTIA SILVAE SINICAE, 1(1): 38-54

Chen W., Gong L., Guo Z.L., Wang W.S., Zhang H.Y., Liu X.Q., Yu S.B., Xiong L.Z., and Luo J., 2013, A novel integrated method for large-scale detection, identification, and quantification of widely targeted metabolites: application in the study of rice metabolomics, Mol. Plant, 6(6):1769-1780 https://doi.org/10.1093/mp/sst080 PMid:23702596

Cai N.H., Deng L.L., Xu Y.L., Xu Y., Zhou L., Wang D.W., Tian B., and Sessa W.C., 2010, A new way to lower blood pressure: pass the chili peppers please!, Cell Metabolism, 12(2): 109-110 https://doi.org/10.1016/j.cmet.2010.07.005 PMid:20674855

Chen W., Gong L., and Guo Z.L., 2013, A Novel Integrated Method for Large-Scale Detection, Identification, and Quantification of Widely Targeted Metabolites: Application in the Study of Rice metabolomics, Molecular plant, 17: 69-80

Guichuan Suo, 2011, Survey results of main vegetable varieties-pepper, China Vegetables, 1(9): 30-32

Kanehisa M., Araki M., Goto S., Hattori M., Hirakawa M., Itoh M., Katayama T., Kawashima S., Okuda S., Tokimatsu T., and Yamanishi Y., 2008, KEGG for linking genomes to life and the environment, Nucleic. Acids. Res. 36(Database issue): D480-D484 https://doi.org/10.1093/nar/gkm882 PMid:18077471 PMCid:PMC2238879

Li J.J., Han S.H., Ding X.L., He T.T., Dai J.Y., Yang S.P., and Gai J.Y., 2015, Comparative transcriptome analysis between the cytoplasmic male sterile line NJCMS1A and its maintainer NJCMS1B in soybean (Glycine max (L.) Merr.), PLoS. One, 10(5): e0126771 https://doi.org/10.1371/journal.pone.0126771 PMid:25985300 PMCid:PMC4436259

Li W.M., Gong J., Bai G.Q., Zhao X.Y., Li S.F., Chen H., AND Cong X.F., 2020, Transcriptome Analysis for Lilium brownii Based on High Throughput Sequencing, Molecular Plant Breeding, 04-09

Li J.J., Ding X.L., Han S.H., Zhang H., Yang L.S., Yang S.P., and Gai J.Y., 2016, Differential proteomics analysisto identify proteins and pathways associated with male sterility of soybean using iTRAQ-based strategy, Proteomics, 138: 72-82 https://doi.org/10.1016/j.jprot.2016.02.017 PMid:26921830 
Molecular Plant Breeding 2020, Vol.11, No.24, 1-8

http://genbreedpublisher.com/index.php/mpb

Liao L.J., 2017, Transcriptomic study on pollen abortion of CMS pepper (Capsium annuum L.), thesis for M.S., Hunan Normal University, Supervisor: Qiu Y.L., $28-44$

Limin Sun, 2019, Analysis of transcription, protein and metabolic regulation mechanisms of flavonoids synthesis in Ginkgo biloba L. leaves, Shandong Agricultural University Ph.D.DISSERTATION, 2019-05-21

Qin Y.L., Shen L.B., and Cao Z.M., 2020, Transcriptome Analysis of Fruits During the Different Developmental Stages in Capsicum annum, Molecular Plant Breeding, 01-03

Roberts A., Pimentel H., Trapnell C., and Pachter L., 2011, Identification of novel transcripts in annotated genomes using RNA-Seq, Bioinformatics, 27(17): $2325-2329$

https://doi.org/10.1093/bioinformatics/btr355

PMid:21697122

Sessa W.C., 2010, A new way to lower blood pressure: pass the chili peppers please! Cell Metab., 12(2): 109-110 https://doi.org/10.1016/j.cmet.2010.07.005 PMid:20674855

Trapnell Cole; Williams Brian A; Pertea Geo; Mortazavi Ali; Kwan Gordon; van Baren Marijke J; Salzberg Steven L; Wold Barbara J; Pachter Lior; 2010, Transcript assembly and quantification by RNA-Seq reveals unannotated transcripts and isoform switching during cell differentiation, Nature biotechnology, 28(5): 511-515

https://doi.org/10.1038/nbt.1621

PMid:20436464 PMCid:PMC3146043

Trapnell C., Williams B.A., Pertea G., Mortazavi A., Kwan G., van Baren M.J., Salzberg S.L., Wold B.J., and Pachter L., 2010, Transcript assembly and quantification by RNA-Seq reveals unannotated transcripts and isoform switching during cell differentiation, Nat Biotechno, 128(5): 511-515

https://doi.org/10.1038/nbt.1621

PMid:20436464 PMCid:PMC3146043

Xuejun Chen. al., 2011, Present situation and Prospect of pepper breeding in China, Modern Horticulture, 6: 44-46

Xianlong Ding, Xuan Wang, Qiang Li, Lifeng Yu, Qijian Song, and Junyi Gai, 2019, Metabolomics Studies on Cytoplasmic Male Sterilityduring Flower Bud Development in Soybean,International Journal of Molecular Science. Sci. 20, 2869 https://doi.org/10.3390/ijms20122869

PMid:31212804 PMCid:PMC6627938 